\title{
COMPUTER GENERATION OF HUMAN GAIT KINEMATICS*+
}

\author{
M. Y. ZARRUGH $\ddagger$ and C. W. RADCLIFFES \\ Department of Mechanical Engineering, University of Michigan, \\ Ann Arbor, MI 48109, U.S.A. \\ and \\ Biomechanics Laboratory, Department of Mechanical Engineering, \\ University of California. Berkeley, CA 94720 , U.S.A.
}

\begin{abstract}
The paper describes a computer program that generates absolute motion variables of human gait from predetermined relative motions. Relative displacements are measured over a range of step rates during both free (seif-determined step rate at different speeds) and forced (forced step rate at a constant speed) walking. converted into harmonic coefficients and stored in an array as a function of step rate. Only six variable identifiers need to be specified to compute any absolute variable or its derivatives at any desirable step rate. The paper displays some examples of measured relative motions and reconstituted absolute variables.
\end{abstract}

\section{INTRODUCTION}

Kinematic variables of gait are valuable to both dynamic synthesis and analysis in the studies of human walking. In dynamic analysis, the measured kinematic data of body segments are used to predict forces and moments applied to each isolated segment. Kinematic measurements are needed in synthesis problems for comparison to motions predicted by the synthesis from a known or assumed set of moments and forces. thus providing a means of evaluating the accuracy of the assumed model.

This paper describes a computer program which generates absolute kinematic data from predetermined relative motions. The relative displacements are measured over a range of walking speeds, converted into harmonic coefficients and stored in an array as a function of step rate. Only six variable identifiers need to be specified to compute any desired variable or its derivatives at any desired step rate. The paper also displays examples of the measured relative motions and reconstituted absolute variables.

The number and variety of kinematic measurements of walking are endless, limited only by the facility and ingenuity of the researcher. The choice of a particular method of measurement, however, is dictated by the purpose of the investigation which is reflected in such considerations as the motions to be measured, a sampling rate sufficient to reproduce the desired degree of detail, accuracy of the measurements and ease of instrumentation and data reduction.

\footnotetext{
- Received for publication 22 August 1978.

+ Research support through contract VAV 101 (134) with the Veterans Administration.

$\ddagger$ Assistant Professor in Mechanical Engineering.

$\S$ Professor of Mechanical Engineering.
}

Measurements of absolute gait variables are best accomplished by photography in its various forms. Photographic techniques of motion measurements include interrupted light method, cinematography and optoelectronic recording.

Marey (1895) invented the interrupted light method which, in its ever-improving form, has been used by authors in all of the classic studies on human gail (Carlet et al., University of Califormia), as well as in more recent studies (Müller and Hettinger, 1952b: Drillis, 1958; Murray et al., 1964; Milner et al., 1973). The reader is referred to Glanville and Kreezer's (1937) comprehensive review of the literature on gait measurements prior to 1937.

Motion pictures, when taken of a moving body and of a timer simultaneously, serve as a satisfactory record of absolute motions. This method is widely used because of the simplicity of the data gathering equipment, ease of data collection and lack of encumbrance to the test subject (University of California, 1947; Levens et al., 1948; Liberson, 1965; Sutherland and Hagy, 1972; Cappozzo et al., 1975). During data gathering mirrors are sometimes placed above the subject (Murray et al., 1967a) or below a glass walkway (Ryker, 1952) at a $45^{\circ}$ angle to obtain multiple views on the same photographs. Three dimensional displacements can also be recorded with stereophotography (Ayoub et al., 1970). In a medical setting, motion picture photography with a stationary or a moving camera is advantageous over other methods especially when the data reduction is mechanized through the use of a motion analyzer (Sutherland and Hagy, 1972).

Optoelectronic recording of motion, a relative newcomer to gait studies, includes television recording and photodetector plate methods. The first method is closely related to cinematography, except that the 
motions are recorded on Videotape instead of film (Winter et al., 1947a and 1974b; Cheng, 1974). The method becomes effective when sophisticated translating equipment are used to reduce the data directly into coordinates (Winter et al., 1972). The photodetector plate method (Selspot), still in the test stage, converts the images of points of interest directly into electrical signals indicating planar position (Lindholm, 1974; Öberg, 1974).

String transducers are a simple and direct means of measuring absolute displacements on the treadmill. The data are obtained in finished form requiring a minimum of further reduction. The main disadvantage is that as many strings as displacements must be attached to the subject. The type of motion measured depends on what instrument is linked to the other end of the string. Linear displacements (Ralston and Lukin, 1969; Lamoreux, 1971; Waters et al., 1973), velocity (Drillis, 1958) and acceleration can be measured.

The body accomplishes its essentially forward displacement by means of a highly coordinated series of rotations of lower limb segments. There are two common methods of measuring body rotations in walking, goniometry and pin studies. A goniometer is a device to measure relative angular rotation between two body segments. To obtain accurate measurement of rotation about a joint, it is necessary to align precisely the measurement axis with the anatomical axis and to insure that the goniometer does not restrict the range of joint rotation. Lamoreux (1974) developed the self-aligning goniometer in which a parallelogram linkage spans a joint allowing rotations to be measured without interference with the joint motion and without the need for axis alignment. He used the method extensively in his complete and systematic kinematic study of gait $(1970,1971)$. Other studies presented segmented goniometric measurements of a few joints with little attention to the effect of speed or axis alignment (Karpovitch et al., 1959 ; Gollnick et al., 1964 ; Wright et al., 1964 ; Finley et al., 1969 ; Johnston and Smidt, 1969; Chao et al., 1970; Kettelkamp et al., 1970;Smidt 1971 ; James and Öberg, 1973; Milner and Dall, 1973). Townsend et al. (1977) described a sixdegree of freedom goniometer to measure the total motion of the knee.

When it is very difficult to attach a measuring instrument onto a segment (e.g. the vertebral column), or more accuracy is needed, pins are inserted directly into the bone, mechanically linking it to a measuring device (Close and Inman, 1953 ; Kinzel et al., 1972b) or to prominent targets for photography (Levens et al., 1948).

Measurements of velocity and acceleration are useful for comparison to derivatives computed from measured displacements. Numerical differentiation, without smoothing or filtering, preferentially magnifies high frequency noise, but careful filtering (Winter et al., 1974a; Pezzack et al., 1977) overcomes the problem. Many investigators measured the vertical and horizontal components (Liberson et al., 1962; Gage, 1967), horizontal component (Waters et al, 1973) and all three components including the lateral (Cavagna et al., 1963; Gersten et al., 1969) component of the acceleration of a point on the trunk approximating the center of mass of the body to compute an estimate of the total force between the feet and the ground. Liberson (1965) measured angular acceleration of the shank for correlation with phasic activities of major hip joint muscles. Accelerometry studies emphasize the frequency spectra of acceleration to distinguish between normal and pathological gait (Gage, 1967; Smidt et al., 1977). Morris (1973) presented a technique using a specially built strain gauge accelerometer to describe the total motion of the shank.

\section{MODELING OF THE WALKING MECHANISM}

\section{Structure}

Analysis in this paper is based on a model of the human skeleton consisting of seven articulated segments as suggested by Elftman (1967). It includes three fundamental divisions : right and left lower limb linked together by a composite segment, the HAT (head-arms-trunk). Each lower limb is divided into a thigh, shank and foot-shoe combination. In all, the model consists of 12 links, six of which comprise the HAT : pelvic girdle, thorax and head, right arm, right forearm, left arm and left forearm. Figure 1 shows a schematic representation of this model in which each segment is identified with a number starting at the pelvis and proceeding distally on the right side and then the left. The assumption of symmetry between right and left sides permits the determination of gait variables of the left side from those of the right side by appropriate shift along the time axis.

\section{Mass and Geometric Parameters}

Despite voluminous published anthropometric data, those of Braune and Fischer (1889) remain the most useful and complete. They expressed anthropometric parameters as ratios or coefficients, so that

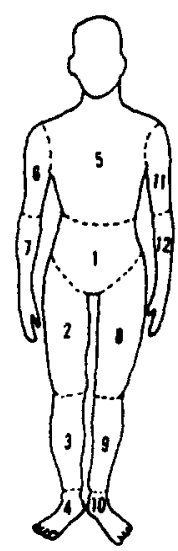

Fig. 1. Schematic representation of body structure. 
Table 1. Approximate coordinates of the centers of mass and joints in the reference position

\begin{tabular}{|c|c|c|c|c|c|}
\hline \multirow[b]{2}{*}{ Segment } & \multirow[b]{2}{*}{$\begin{array}{l}\text { Segment } \\
\text { No. }\end{array}$} & \multirow{2}{*}{$\begin{array}{l}\text { Mass } \\
\text { ratio }\end{array}$} & \multicolumn{3}{|c|}{ Coordinates relative to height } \\
\hline & & & $\begin{array}{c}\text { Horizontal } \\
x\end{array}$ & $\begin{array}{c}\text { Vertical } \\
y\end{array}$ & $\begin{array}{c}\text { Lateral } \\
z\end{array}$ \\
\hline HAT* $^{*}$ & 0 & 0.680 & 0.0 & 0.644 & 0.0 \\
\hline Pelvis & 1 & 0.278 & 0.0 & 0.560 & 0.0 \\
\hline Thigh & 2 & 0.100 & -0.0024 & 0.420 & 0.047 \\
\hline Shank & 3 & 0.045 & -0.0016 & 0.178 & 0.043 \\
\hline Foot & 4 & 0.015 & 0.0100 & 0.018 & 0.051 \\
\hline Hip & - & - & 0.0 & 0.527 & 0.049 \\
\hline Knee & - & - & 0.0 & 0.300 & 0.045 \\
\hline Ankle & - & - & -0.1070 & 0.042 & 0.041 \\
\hline
\end{tabular}

* Includes head, arms and the trunk.

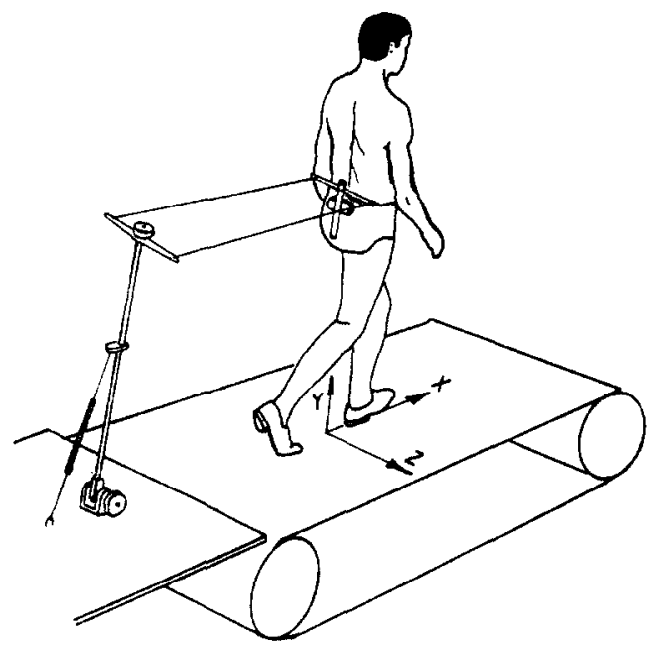

Fig. 2. Definition of the coordinate system.

differences between individuals were taken into account. Their data on center of mass and approximate joint locations are used in this study. As tabulated in Table 1, these three dimensional coordinates (see Fig. 2 for a definition of the coordinate system) are expressed as ratios of height without shoes. Only parameters of the right limb are shown, because left to right symmetry is assumed. Parameters expressing the distribution of mass in the body are obtained from Braune and Fischer's data or computed from geometrical approximations of segments as truncated ellipsoidal cones, using Dempster's data (1955) for establishing the major and minor axes of the conical cross-sections. Table 1 lists the ratio of segment masses relative to

Table 2. Subject's personal data

\section{Age, yr}

Body mass, barefoot, no instruments, $\mathrm{kg}_{\mathrm{g}}$ 54

Body mass with shoes and instruments, $\mathrm{kg}$

Shoes mass, kg

Height above floor without shoes, $\mathrm{cm}$

Shoe heel height. $\mathrm{cm}$

Shoe length, cm total body mass without shoes. The personal data of the subject of this study are tabulated in Table 2.

\section{RELATIVE DISPLACEMENT MEASUREMENTS}

\section{Instrumentation and data gathering}

The system of measurements (Fig. 3 ) as designed by Lamoreux (1970), is used to locate the pelvic girdle in space via its six degrees of freedom and then to measure the rotations of each lower limb segment relative to the one proximal to it, starting with measurements of thigh rotations relative to the pelvic girdle. Locating the pelvis in space is accomplished by three pairs of parallel strings connecting the pelvic girdle to spring-loaded instrumented arms (Fig. 2) above, behind and to the right of the subject. For the sake of simplicity, only the rear arm, measuring the forward displacement of the pelvis and its rotation about a vertical axis, is shown in Fig. 2. Joint rotations are measured by potentiometers (Cummings and Lamoreux, 1972) attached to segments by means of

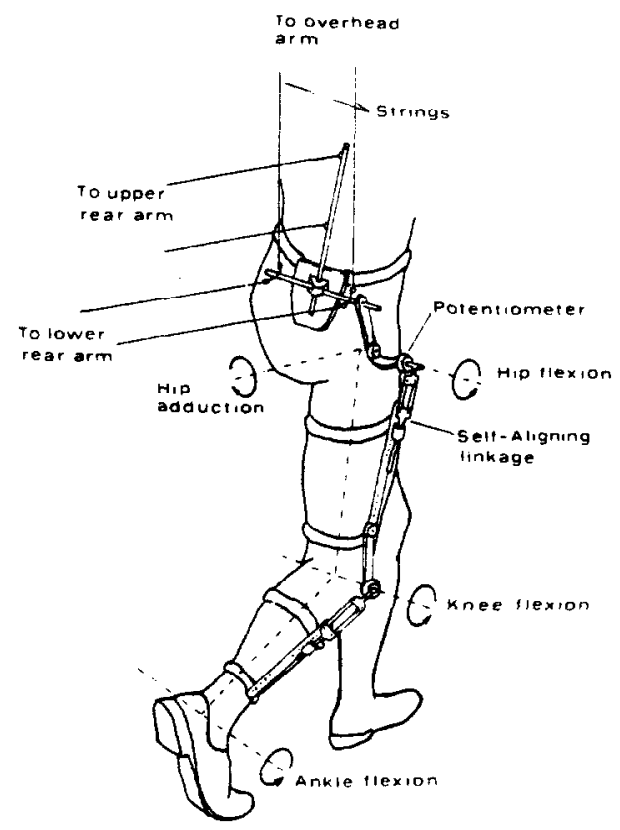

Fig. 3. System of motion measurement. 
light aluminum frames and spanning the joints with self-aligning linkages (Lamoreux, 1974). In addition to the six displacements of the pelvis, hip, knee and ankle flexion angles are measured. Hip adduction is interpolated from Lamoreux's (1970) data to match the current set of measurements. Data during sixteen full walking cycles measured from right heel contact to the next right heel contact, are sampled at a rate of 200 samples per sec and stored in digital form. The heel contact marks are generated by microswitches attached to the heels of both right and left shoes. The heel events data as well as the treadmill displacement in millimeters are also recorded.

Kinematic data during both free (self-selected step rate) and forced (forced step rate) walking are collected. The free pattern data are gathered over a range of treadmill speeds corresponding to a step range of $85-130 \mathrm{steps} / \mathrm{min}$ at $5 \mathrm{steps} / \mathrm{min}$ intervals. In the forced pattern experiment, the step rate was forced on the subject via an electronic metronome within a step rate range of $90-140 \mathrm{steps} / \mathrm{min}$ at a constant speed of 90 $\mathrm{m} / \mathrm{min}$. To overcome subject dependent random variations, all gathered data are averaged over sixteen cycles after reviewing for consistent features. Data processing and storage are facilitated by compressing the data into harmonic coefficients form. A fast Fourier transform program converted all measured variables into harmonic coefficients. Seven to twelve harmonics are used to reconstruct all gait variables, except ankle flexion which required at least 20 harmonics for proper reconstitution.

\section{RESULTS}

\section{Step length and step rate}

This basic gait pattern has been shown (Zarrugh et al., 1974) to be highly related to metabolic energy expenditure. Its influence on the kinematic aspects of gait are discussed elsewhere by Lamoreux and Todd (1974). They showed that kinematic measurements on the same subject are highly repeatable if they were made under the same conditions of step length and frequency. However, varying the speed, step rate $(n)$ or step length $(s)$ had a profound effect on the measured motions. Therefore, it is necessary to accompany any measured kinematic data by the associated data on average walking velocity, step rate and step length.

The $s-n$ pattern data for our subject are displayed in Fig. 4 in which the solid line depicts the constant $s$ to $n$ ratio fit and the interrupted line displays the constant slope relationship $\left(s=c_{0}+c_{1} n\right)$. A comparison between these two forms in terms of the sum of squared errors shows a slightly smaller sum $\left(38.5 \mathrm{~cm}^{2}\right)$ or slightly better fit, for the constant $s-n$ ratio form than the constant slope relationship which has an error sum of $50.3 \mathrm{~cm}^{2}$.

\section{Free pattern kinematic data}

Each of the measured kinematic displacements is presented as a family of curves, one for each tested step rate (or speed), as a function of time in percent of cycle to facilitate visualization of speed effect (Fig. 5a-i). The corresponding harmonic coefficients are available

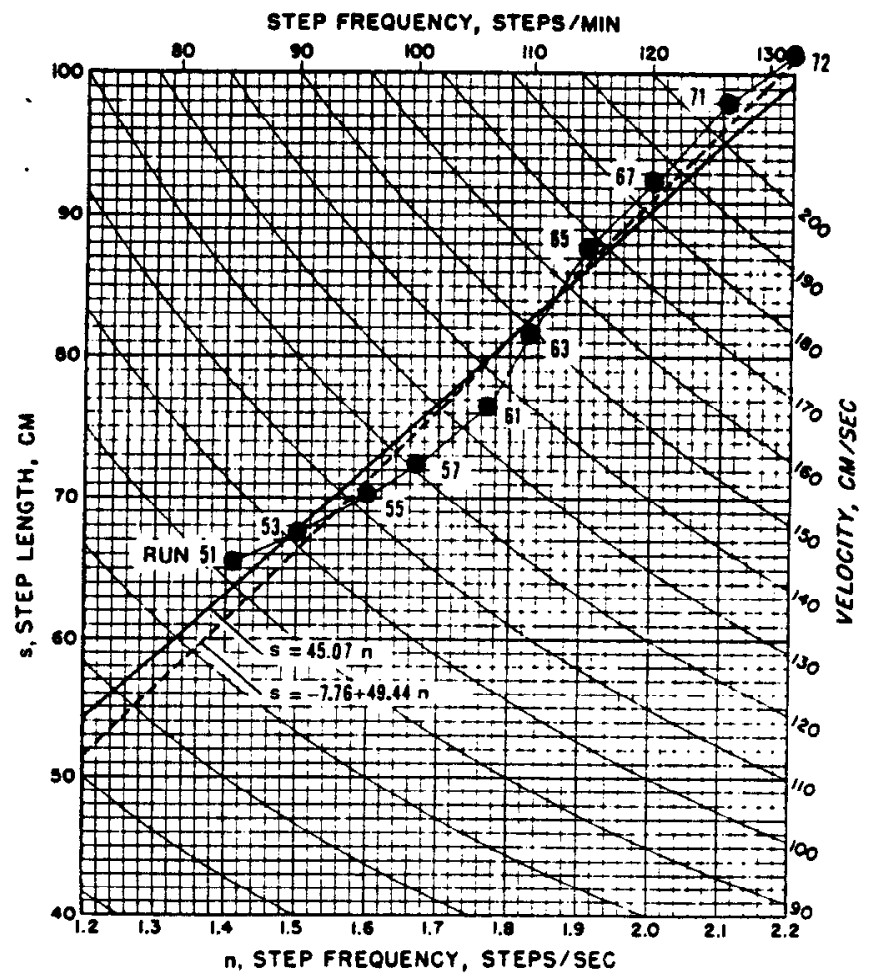

Fig. 4. Step length, step rate and velocity measures. 

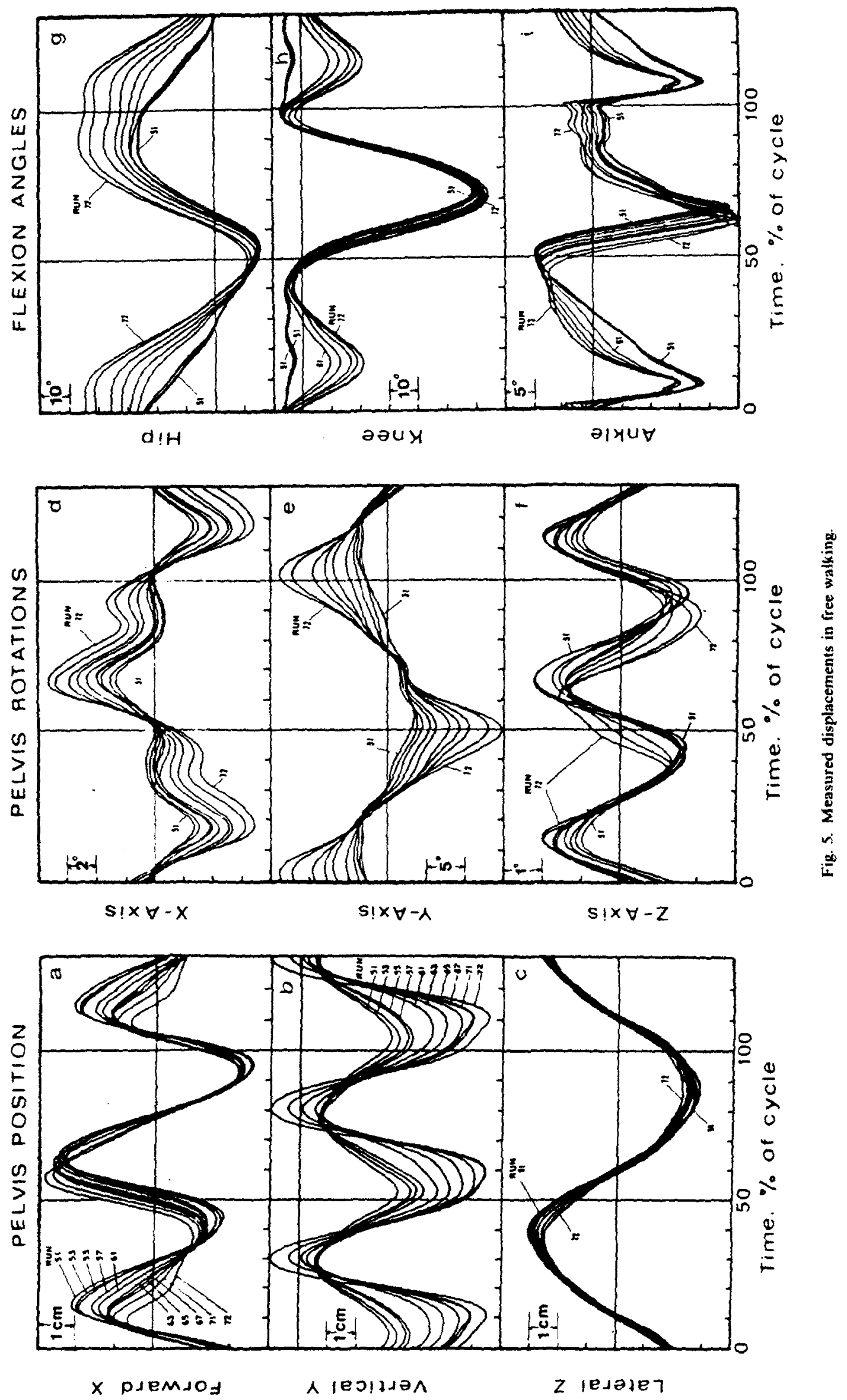

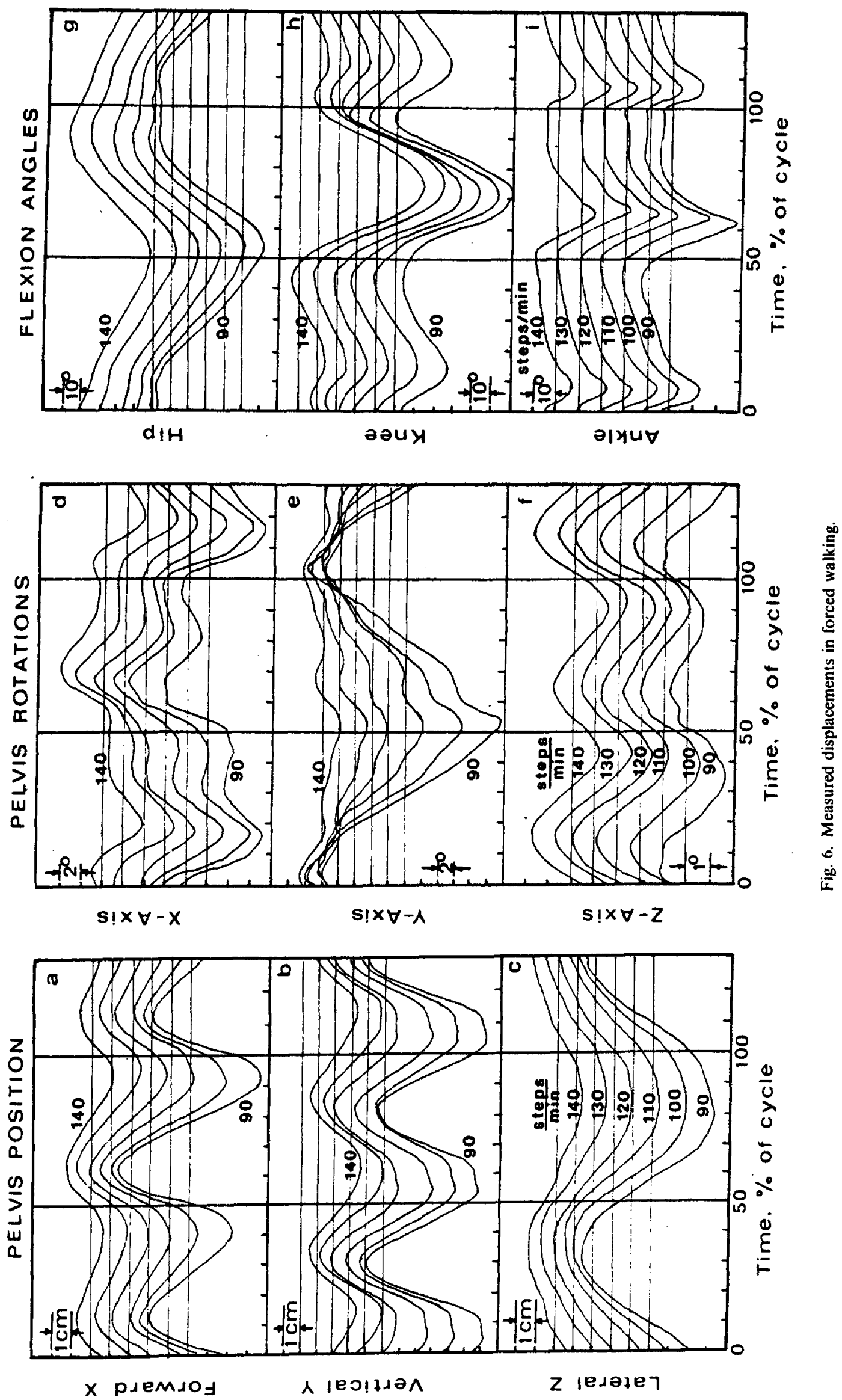
from the authors. The hip adduction angle data were interpolated from Lamoreux (1970).

One of the remarkable features displayed in this data is the abrupt change in the flexion patterns of the hip, knee and ankle joints occurring at about 105 steps/min. Below this transitional step rate, in the range of $85-100$ steps $/ \mathrm{min}$, the flexion patterns for each joint are almost identical showing almost no effect due to increased step rate. Within the same step rate range, the knee remains almost fully extended (Fig. 5h) during most of stance phase $(0-40 \%)$. Maximum ankle extension which normally occurs shortly (less than $1 \%$ cycle) before toe-off is observed in Fig. $5 \mathrm{i}$ to take place essentially at the same time $(66 \%$ cycle). Increased step rates above the transitional one result in increased knee flexion during stance phase (Fig. 5h), earlier and earlier toe-off points (Fig. Si) and increased maximum hip flexion (Fig. $5 \mathrm{~g}$ ).

\section{Forced pattern data}

In order to investigate the conditions under which the body minimizes total work done during locomotion, it is necessary to force combinations of step length and step rate which are not adopted normally in gait. This is accomplished by keeping the speed constant at $90 \mathrm{~m} / \mathrm{min}$, while imposing a set of varying step rates between 90 and 140 steps $/ \mathrm{min}$. Figures $6 \mathrm{a}-\mathrm{c}$ display the measured kinematic variables for this pattern of walking. The harmonic coefficients for the same data are available from the authors.

Despite constant speed, an observable effect is shown of the changing step length on each measured variable. This dramatically illustrates how the basic gait parameters, speed, step length and step rate, influence the kinematic variables of walking. Lower limb flexion angles (Figs. 6a-i) are not greatly affected

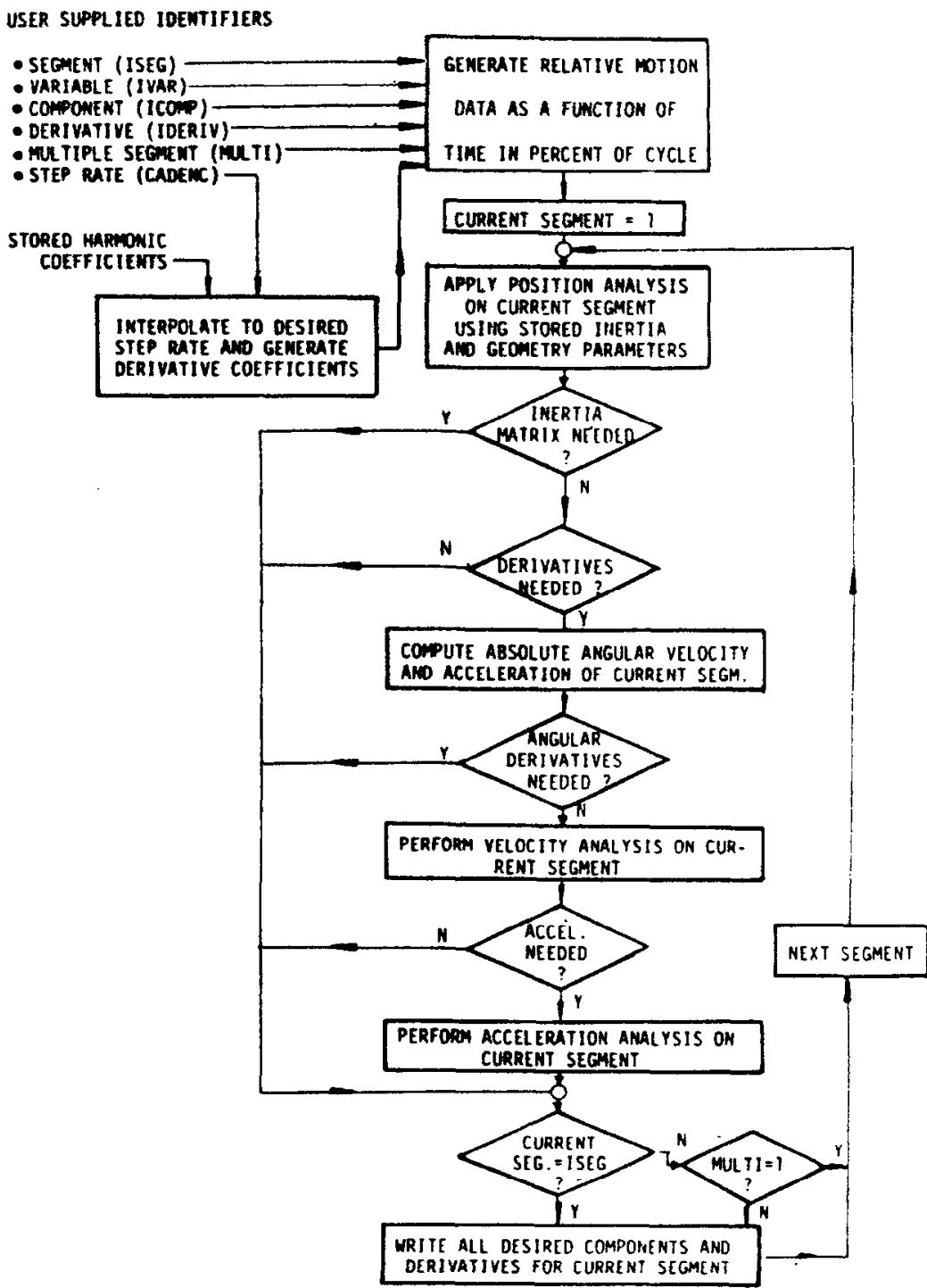

Fig. 7. Simplified flow chart of the method of solution for absolute motions from measured displacements. 
by change in step length. In contrast, the step length has the largest influence on pelvic displacements, especially on the three linear displacements (Figs. $6 \mathrm{a}-\mathrm{c}$ ) and the rotation about a vertical axis (Fig. 6e) which are observed to increase in amplitude with increased step length.

\section{ABSOLUTE COORDINATES DETERMINATION}

\section{Kinematic analysis}

For the purpose of dynamic analysis of gait, the human body can be treated as a chain of rigid links interconnected at joints which allow the links to rotate relative to each other. The system of successive relative measurements starts with defining the position of the trunk relative to the chosen fixed coordinate system (Fig. 2) and proceeds to express the rotation of each lower limb segment relative to the one proximal to it. The computation of the absolute motions takes a similar course; it begins with computing the absolute position of the trunk as a reference member and continues distally to describe the absolute motion of all segments.

An analytical method of computing absolute motions from relative measurements is developed in Appendix A for a simple chain with two links rotating relative to each other about three axes. Figure 12 depicts such a general linkage consisting of a proximal link $(i-1)$, the motion of which is assumed to be known completely and a distal link (i) the motion of which is to be found. The relative rotations are measured successively at the current orientation of the instrument axes (Fig. 12). The final configuration of any line on the link is determined in terms of a rotation matrix that takes into account all successive relative rotations (Appendix A). The velocity and acceleration analyses of the two-link chain are developed in terms of the first and second derivatives computed from the relative measured joint rotations as shown in Appen$\operatorname{dix} \mathbf{A}$.

\section{Method of solution}

The kinematic analyses of the previous section, developed for a general link, can be applied successively to the entire system of twelve links. For this purpose a program is written in FORTRAN IV that computes absolute motions of these links from internally stored harmonic coefficients. To simplify use, the user need supply only six parameters identifying the desired gait variability. The first four parameters define the variable. The fifth parameter is a multiple segment flag designed to save processing time if data of many interconnected segments are needed. The sixth defines the step rate.

The method of computation in a simplified form is displayed in Fig. 7. On the basis of the user's six identifiers, the program interpolates the harmonic data of the necessary measured variables to the specified step frequency, generates derivative coefficients if necessary and reconstitutes the harmonic data into equivalent time functions. If only measured variables are requested, no further processing is done;

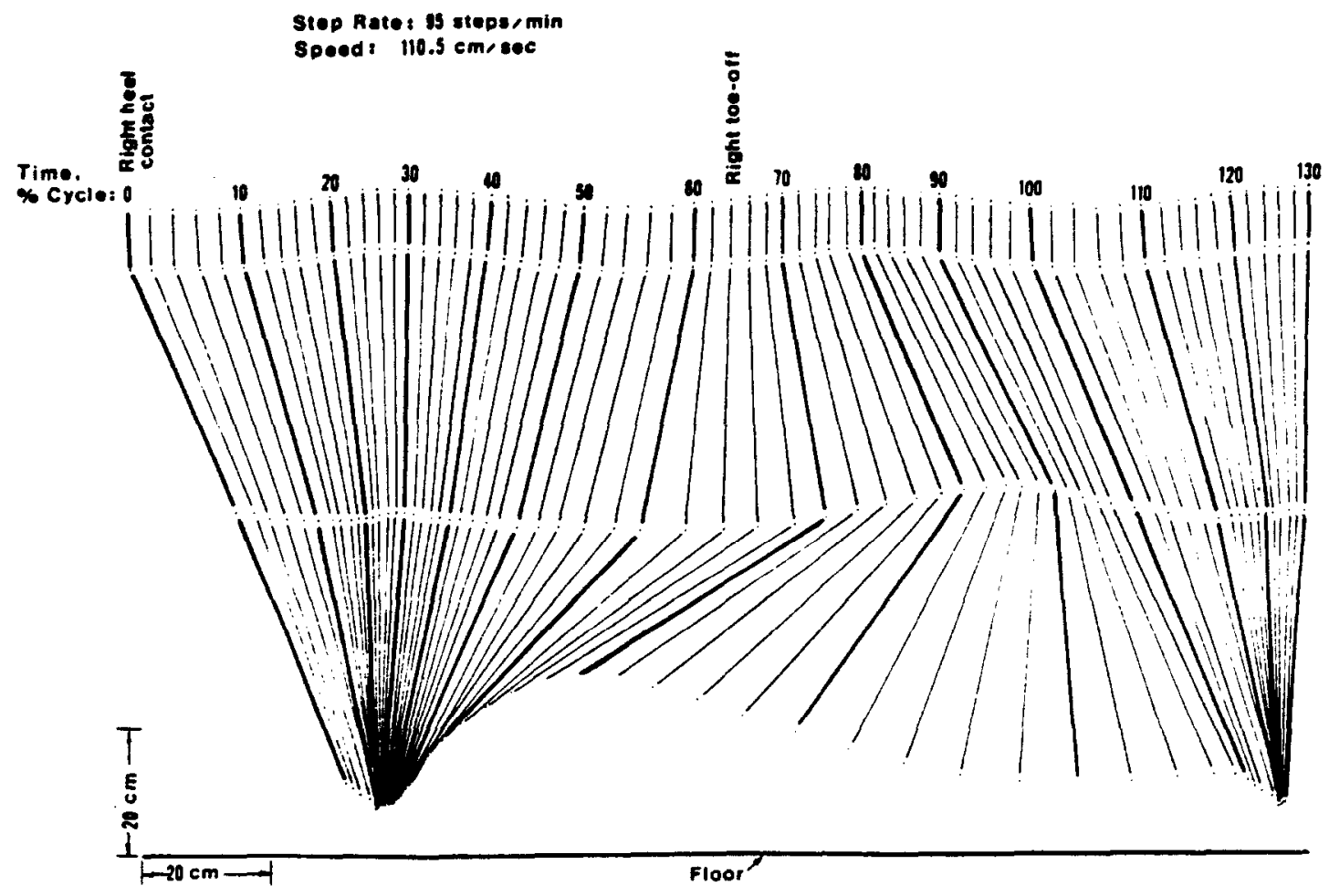

Fig. 8. Stick diagram of the subject walking at $95 \mathrm{steps} / \mathrm{min}$. 


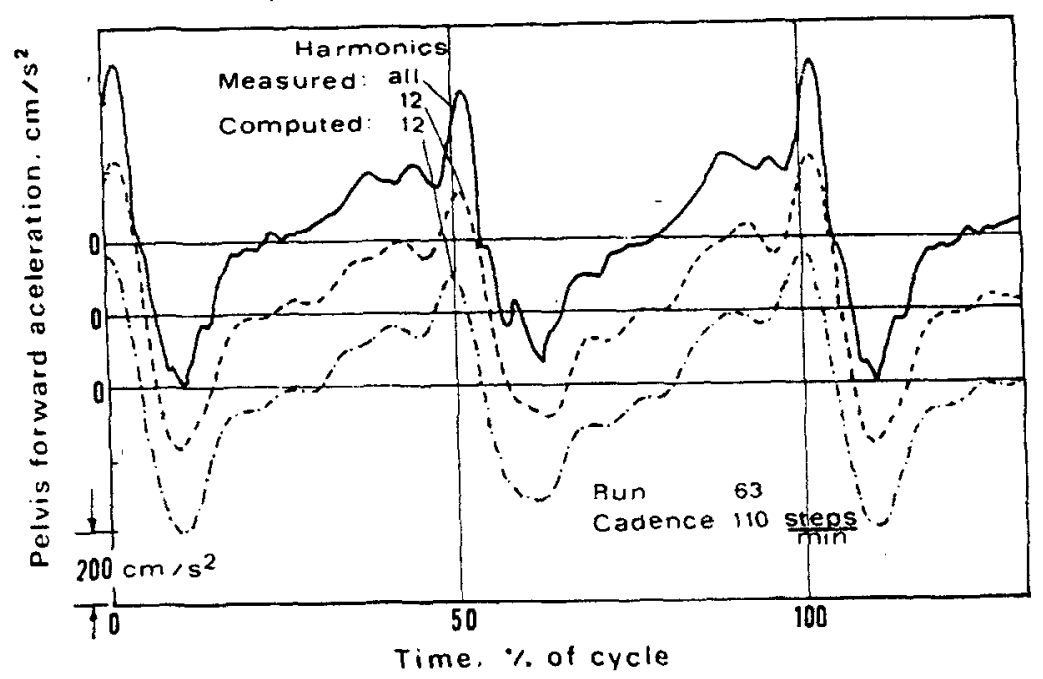

Fig. 9. Measured and computed forward peivic accelerations.
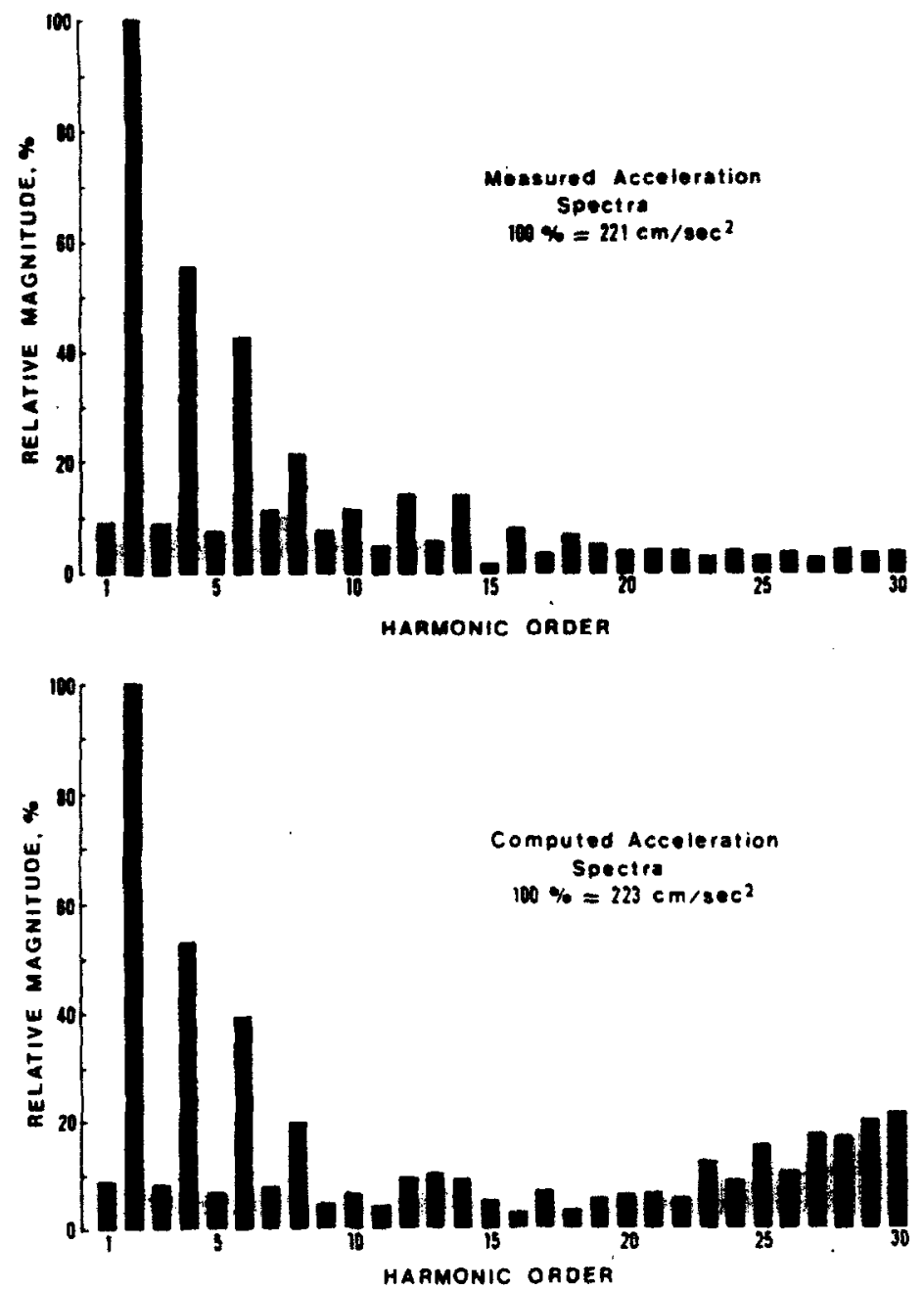

Fig. 10. Harmonic spectra of forward pelvic accelerations. 


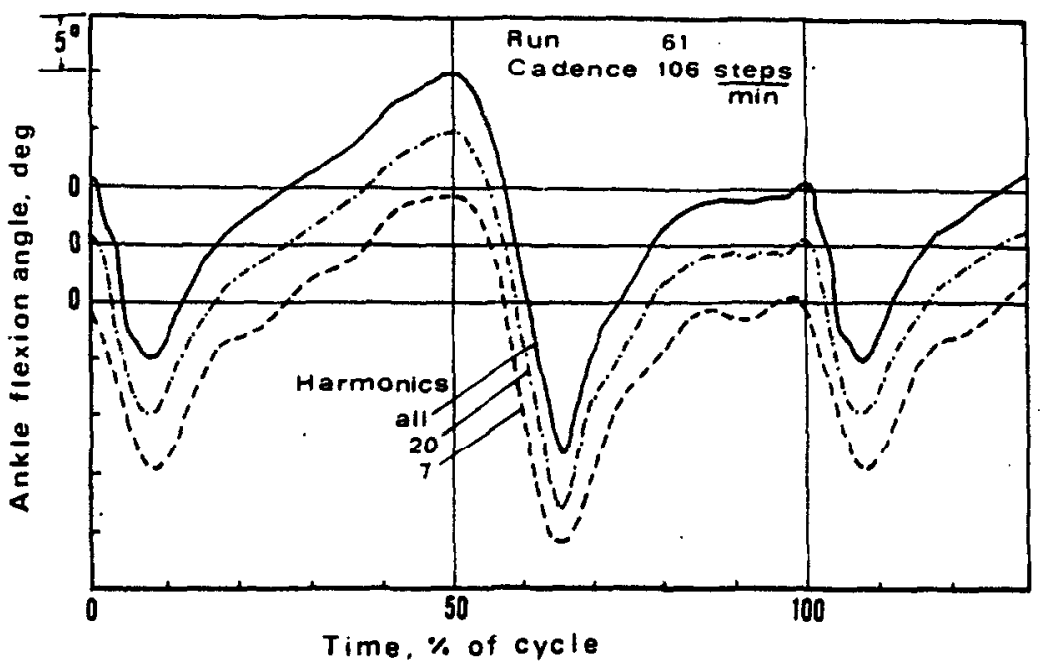

Fig. 11. Effects of harmonic content on the reconstitution of ankle flexion as a time function.

otherwise the program performs kinematic analyses as necessary until all requests are satisfied.

\section{Samples of computed variables}

Although mass center and joint coordinates of all segments can be displayed, a "stick" diagram is chosen for display rather than the coordinates because it provides a clear and brief illustration of absolute motions in the plane of progression. Figure 8 displays such a diagram of our subject at a step rate of 95 steps $/ \mathrm{min}$ in which the stick representing the foot is deleted for clarity (compare to Fig. 15.4 in Eberhart et al., 1954a).

An evaluation of the numerical harmonic differentiation is made in Fig. 9 in which the experimental and computed (from displacement) horizontal pelvic accelerations are displayed as a function of time in percent of cycle. Two measured acceleration curves are shown: one includes all harmonics and the other the lower twelve harmonics. The corresponding harmonic spectra are depicted in Fig. 10 which indicates that the harmonic content is concentrated in the low frequency range. This is supported in other studies, such as Winter et al. (1974a) and Cappozzo et al. (1975). At high frequencies (above the fifteenth harmonic), the spectra resemble those of white noise which are characterized by uniform magnitude over all frequencies. Because proportionately smaller amounts of information and larger amounts of noise are added if a large number of harmonics are used, seven to twelve harmonics are considered a good compromise between information and noise for most gait variables, except ankle flexion which requires at least 20 harmonics for adequate reconstruction. Figure $11 \mathrm{com}$ - pares ankle flexion curves obtained by direct measurement, harmonic synthesis with 20 harmonics and synthesis with seven harmonics. Some degree of detail is lost when fewer harmonics are used.

\section{REFERENCES}

Ayoub, M. A., Ayoub, M. M. and Ramsey, J. D. (1970) A stereometric system for measuring human motion. Human Factors 12(6), 523-535.

Berstein, MN. A. (1967) The Coordination and Regulation of Movements. Pergamon Press, Oxford.

Braune, W. and Fischer, O. (1889) Uber den den Schwerpunkt des menschlichen Körpers, mit Rücksicht auf die Aüstrustung des deutschen Infanteristen. (On the center of gravity of the human body as related to the equipment of the German infantry.) Abh. sächs. Gesellschaft Wissenschaften 26, 561-672.

Cappozzo, A.. Leo. T. and Pedotti, A. (1975) A general computing method for the analysis of human locomotion. J. Biomechanics 8, 307-320.

Carlet, M. G. (1872) Essai experimentale sur la locomotion humaine. (Experimental essay on human locomotion.) Annls Sci. nat. 16, 1-92.

Cavagna, G. A., Saibene, F. P. and Margaria, R. (1963) External work in walking. J. appl. Physiol. 18, 1-9.

Chao, E. Y.-S., Rim, K., Smidt, G. L. and Johnston, R. C. (1970) The application of $4 \times 4$ matrix method to the correction of measurements of hip joint rotations. $J$. Biomechanics 3, 459-471.

Cheng, I.-S. (1974) Computer-Television Analysis of Biped Locomotion. The Communication and Control Systems Laboratory, Department of Electrical Engineering, Ohio State University, Columbus, Ohio.

Close, J. R. and Inman, V. T. (1952) The Action of the Ankle Joint. Prosthetic Devices Research Project, Institute of Engineering Research, University of California. Berkeley.

Cummings, R. W. and Lamoreux, L. W. (1972) The use of electrical transducers in the measurement of body motions. Bull. Prosthetics Res. 10-18, 46-59. 
Dempster. W. F. (1955) Space requirements of the seated operator. WADC Technical Report 55-159. Wright Air Development Center, United States Air Force, WrightPatterson Air Force Base, Ohio.

Drillis, R. (1958) Objective recording and biomechanics of pathological gait. Ann. N.Y. Acad. Sci. 74, 86-109.

Eberhart, H. D., Inman, V. T. and Bresler, B. (1954) The principal elements of human locomotion. In Human Limbs and Their Substitutes. (Edited by P. E. Klopsteg and P. D. Wilson et al.J, pp. 437-471. McGraw-Hill, New York.

Elftman, H. (1967) Basic function of the lower limb. Biomed. Engng 2, 342-345.

Finley, F. R., Cody, K. A. and Finizie, R. V. (1969) Locomotion patterns in elderly women. Archs phys. Med. Rehabil. 50, 140-146.

Fischer, O. (1895-1904) Der Gang des Menschen. Abhandlungen der mathematischen Klasse der KöniglichSächischen Gesellschaft der Wissenchaften. 8 vols. (Vol. 1 coauthored by W. Braune).

Gage, H. (1967) Accelerographic analysis of human gait. In Biomechanics Monograph, pp. 137-152, A. S. M. E., New York.

Gersten, J. W., Orr, W., Sexton, A. W. and Okin, D. (1969) External work in level walking. J. appl. Physiol. 26, 286-289.

Glanville, A. D. and Kreezer, G. (1937) The characteristics of gait of normal male adults. J. exp. Psychol. 21, 277-301.

Gollnick, P.D. and Karpovich, P. V. (1964) Electrogoniometric study of locomotion and of some athletic movements. Res. Quart. 35, 357-369.

James, U. and Oberg, K. (1973) Prosthetic gait pattern in unilateral above-knee amputees. Scand. J. rehabil. Med. 5 , $35-50$.

Johnston, R. C. and Smidt, G. L. (1969) Measurement of hipjoint motion during walking: Evaluation of an electromyographic method. J. Bone Jt Surg. (A)51, 1083-1094

Karpovich, P. V. and Wiklow, L. B. (1959) A goniometric study of the human foot in standing and walking. U.S.A.F. med. J. 10, 885-903.

Kettelkamp, D. V., Johnson, R. J., Smidt, G. L., Chao, E. Y.-S and Walker, M. (1970) An electrogoniometric study of knee motion in normal gait. J. Bone Jt Surg. (A)52, 775-790.

Kinzel, F. L., Hillberry, B. M., Hall, A. S., Jr, Van Sickle, D. C and Harvey, W. M. (1972) Measurement of the total motion between two body segments-II. Description of application. J. Biomechanics 5, 283-293.

Lamoreux, L. W. (1970) Experimental kinematics of human walking. Dissertation Ph.D., Mechanical Engineering. University of California, Berkeley.

Lamoreux, L. W. (1971) Kinematic measurement in the study of human walking. Bull. Prosthetics Res. BPR 10-15, 3-84.

Lamoreux, L. W. (1974) A self-aligning goniometer for simplified gait analysis. Proc. World Congr. of ISPO (Int Soc. Prosthetics and Orthotics), 8-12 Oct. Montreux. Switzerland

Lamoreux, L. W. and Todd. F. N. (1974) The importance of time and distance measurements for analysis of human walking. Proc. World Congr. ISPO (Int. Soc. Prosthetics and Orthotics), 8-12 Oct. Montreux, Switzerland.

Levens, A. S., Inman, V. T. and Blosser, H. A. (1948) Transverse rotation of the segments of the lower extremity in locomotion. J. Bone Jt Surg. (A) 30, 859-972.

Liberson, W. T., Holmquest, H. J. and Halls, A. (1962) Accelerographic study of gait. Arch. phys. Med. Rehabil. 43, 547-554.

Liberson, W. R. (1965) Biomechanics of gait: A method of study. Arch. phys. Med. Rehabil. 46, 37-48.

Lindhoim, L. E. (1974) An optoelectronic instrument for remote on-lise movement monitoring. In Biomechanics IV (Edited by R. C. Neison and C. A. Morehouse), pp. 510-512. University Park Press, Baltimore.

Marey, E. J. (1895) Mocement. Appleton, New York. (Orig- inally published in French by Masson, Paris, 1894.)

Milner, M., Dall, D., McConnell, U. S., Brennan. P. K. and Hershler, C. (1973) Angle diagrams in assessment of locomotion function. S. Afr. med. J. 47. 951-957.

Morris, J. R. W. (1973) Accelerometry - A technique for the measurement of human body movements. J. Biomechunic: 6, 729-736.

Müller, E. A. and Hettinger, Th. (1952) A rbeitsphysiologische Untersuchungen verschiedener Oberschenkel-Kunstbeine. (Energy study of different above-knee prostheses.) $Z$. Orthop. 81, 525-545.

Murray, M. P., Drought, A. V., Ross, C. and Kory, R. C. (1964) Walking patterns of normal men. $J$. Bone Jt Surg. A $(2) 46,335-360$.

Murray, M. P. (1967) Gait as a total pattern of movement. Am. J. phys. Med. 46, 290-333.

Öberg, K. (1974) Mathematical modeling of human gait : An application of SELSPOT-system. In Biomechanics $I V$ (Edited by R. C. Nelson and C. A. Morehouse), pp. 79-84. University Park Press, Baltimore.

Pezzack, J. C., Norman, R. W. and Winter, D. A. (1977) An assessment of derivative determining techniques used for motion analysis. J. Biomechanics 10, 377-382

Pipes, L. A. (1963) Matrix Methods for Enyineering. PrenticeHall, Englewood Cliffs.

Ralston, H. J. and Lukin, L. (1969) Energy levels of human body segments during level walking. Ergonomics 12, 39-46.

Ryker, N. J., Jr. (1952) Glass Walkway Studies of Norma Subjects During Normal Level Walking. Prosthetic Devices Research Project, Institute of Engineering Research, University of California, Berkeley. Series II, Issue 20. Berkeley, The Project.

Smidt, G. L. (1971) Hip motion and related factors in walking J. Am. phys. Therapy Ass. 51, 9-21.

Smidt, G. L., Deusinger, R. H., Arora, J. and Albright, J. P (1977) An automated accelerometry system for gait analysis. J. Biomechanics 10, 367-375.

Suh, C. S. and Radcliffe, C. W. (1967a) Synthesis of plane linkages with use of the displacement matrix. J. Engng Ind. (B) $99,206-214$.

Suh, C. H. and Radclifie, C. W. (1967b) Synthesis of spherical linkages with use of the displacement matrix. J. Engng Ind. (B) $199,215-222$.

Sutherland, D. H. and Hagy, J. L. (1972) Measurement of gait movements from motion picture film. $J$. Bone $J t$ Surg. (A 154, 787-797.

Townsend, M. A., Izak, M. and Jackson, R. W. (1977) Tota motion knee goniometry. J. Biomechanics 10, 183-193.

University of Califomia-Berkeley (1947) Fundamental Studies of Human Locomotion and Other Material Relating to Design of Artificial Limbs. Prosthetic Devices Research Project, Institute of Engineering Research, Final Report to the Committee on Artificial Limbs, National Research Council. 2 Vols. Berkeley, The Project.

Waters, R. L., Morris, J, and Perry, J. (1973) Translational motion of the head and trunk during normal walking. $J$ Biomechanics 6, 167-172.

Winter, D. A., Greenlaw, R. K. and Hobson, D. A. (1972) Television-Computer analysis of kinematics of human Gait. Comp. Biomed. Res. 5, 498-504.

Winter, D. A., Sidwall, H. G. and Hobson, D. A. (1974a) Measurement and reduction of noise in kinematics of Jocomotion. J. Biomechanics 7, 157-159.

Winter, D. A., Quanbury, A. O., Hobson, D. A., Sidwall, H G., Reimer, G., Trenholm, B. G., Steinke, T. and Shlosser. H. (1974) Kinematics of normal locomotion-A statistical study based on T.V. data. J. Biomechanics 7, 479-486.

Wright, D. G., Desai, S. M. and Henderson, W. H. (1964) Action of the subtalar and ankle-joint complex during the stance phase of walking. J. Bone Jt Surg. (A)46, 36)-382

Zarrugh, M. Y., Todd, F. N. and Ralston, H. J. (1974 Optimization of energy expenditure during level walking Eur. J. appl Physial. 33, 293-306. 
APPENDIX A

Spatial Kinematic Analysis

\section{Position analysis}

The rotation matrix (Suh and Radcliffe, 1967a and 1967b) approach is used to determine absolute displacements from relative rotation measurements. The method is developed for a simple two-link chain with a joint $a_{\text {, that }}$ allows three successive rotations $\phi_{w}, \phi_{v}$ and $\phi_{v,}$ about nonparallel axes. This simple chain is represented in Fig. 12. The absolute motion of proximal link $i-1$ is assumed to be completely known from previous kinematic analyses which start at the reference link having a known absolute motion. The purpose of this analysis is to determine the motion of the distal link $i$ in terms of the known absolute motion of the proximal link $i$ -1 and the relative rotations in the joint which are measured about instrument axes $\boldsymbol{u}_{i}, v_{i}$ and $w_{i}$

Having determined the absolute rotation matrix $\left[R_{i-1}\right]$ for member $i-1$, a similar matrix $\left[R_{i}\right]$ can be computed for member $i$ by locating the current orientation of the measurement axes. The initial orientations of these axes are represented by $u_{0 i}, v_{0 i}$ and $w_{0 t}$ and are estimated from photographs of the subject wearing the measurement apparatus while standing in relaxed position. Table 3 lists the direction cosines of these vectors for all joints relative to the reference coordinate system. The axis $u_{f}$ is rigidly attached to the proximal member $i-1$ (Fig. 12), so when this member executes a rotation $\left[R_{1-1}\right]$ from the reference position, the vector $u_{0 i}$ rotates with the member to assume its final orientation $\mathbf{u}_{i}$ :

$$
\mathbf{u}_{i}=\left[R_{i-1}\right] \mathbf{u}_{0 i},
$$

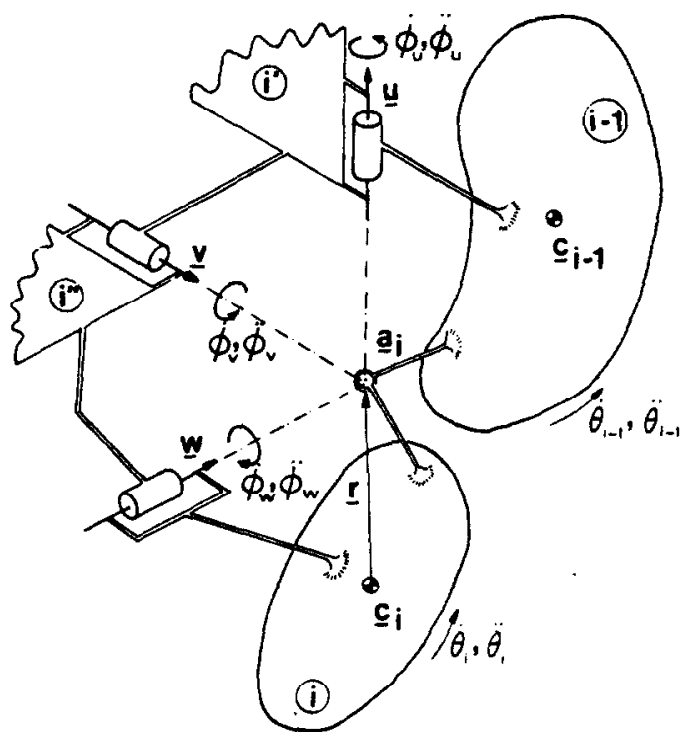

Fig. 12. Two body segments represented as an idealized twolink chain connected through a point $a_{\text {i }}$ by the linkage measuring the relative rotations between them. where

$\left[R_{i-1}\right]=$ absolute rotation matrix of member $i-1$

and

$$
u_{0 t}=\text { initial orientation of measurement axis } \mathbf{n}_{i} \text {. }
$$

The relative rotation matrix $\left[R_{\mathrm{w}}\right]$ can now be constructed to account for measured relative rotation $\phi_{\|}$of member $i$ about u:

$$
\left[R_{u}\right]=\left[\begin{array}{lll}
u_{1}^{2} V+C & u_{1} u_{2} V-u_{3} S & u_{1} u_{3} V+u_{2} S \\
u_{1} u_{2} V+u_{3} S & u_{2}^{2} V+C & u_{2} u_{3} V-u_{1} S \\
u_{1} u_{3} V-u_{2} S & u_{2} u_{3} V+u_{1} S & u_{3}^{2} V+C
\end{array}\right],
$$

where

$$
\begin{aligned}
C & =\cos \left(\phi_{0}\right) \\
S & =\sin \left(\phi_{0}\right) \\
V & =\cos \left(\phi_{0}\right) \\
u_{1}, u_{2} \text { and } u_{3} & =\text { direction cosines } \mathrm{u} .
\end{aligned}
$$

Because axis $v_{t}$ is distal to $u_{l}$, its final orientation is found in terms of rotations of axis $u_{i}$ and member $i-1$ :

$$
v_{i}=\left[R_{i-1}\right]\left[R_{\mathbf{E}}\right] \mathrm{v}_{01} \text {. }
$$

A similar rotation matrix to $\left[R_{\mathrm{u}}\right]$ can be written for relative rotation $\phi_{v}$ about axis $v_{i}$ by replacing $\phi_{v}$ with $\phi_{v}$ in equation (A2). The current direction of third measurement axis $\pi_{i}$ can now be computed in terms of absolute rotation of member $i$ -1 and relative rotations about $u_{i}$ and $v_{i}$ :

$$
w_{i}=\left[R_{i-1}\right]\left[R_{w}\right]\left[R_{v}\right] w_{0 i}
$$

Through the known final configurations of all three measurements axes, the absolute rotation matrix $\left[R_{i}\right]$ for link $i$ is found :

$$
\left[R_{i}\right]=\left[R_{i-1}\right]\left[R_{\mathbf{v}}\right]\left[R_{\mathrm{v}}\right]\left[R_{\mathrm{w}}\right] .
$$

The matrix $\left[R_{w}\right]$ has the same form as equation (A2) except $\phi_{k}$ is replaced by $\phi_{W}$. Although $\left[R_{i}\right]$ is derived for a general joint having three degrees of freedom, only the hip and ankle allow as many rotations while the knee allows only two rotations. Because of small amplitude compared to flexion angles, some of these rotations, such as ankle inversion, knee and ankle axial rotations are ignored in the analysis. With this simplification, the rotations considered in the study are the flexion angles of the hip, knee and ankle joints, as well as hip adduction.

Because point $a_{1}$ is treated as a joint on link $i-1$, its coordinates are determined in the previous stage of analysis on member $i-1$. Therefore, the coordinates of the distal joint $a_{i+1}$ and the center of mass $c_{i}$ on link $i$, are formulated in terms of all known quantites:

$$
\begin{aligned}
c_{i} & =a_{i}+\left[R_{i}\right]\left(c_{0 i}-a_{0 i}\right) \\
a_{i+1} & =a_{i}+\left[R_{i}\right]\left(a_{0 i+1}-a_{0 i}\right),
\end{aligned}
$$

where

$a_{i}=$ distal joint on link $i-1$

$\left[R_{i}\right]=$ absolute rotation matrix of link $i$

$c_{01}=$ initial position of the center of mass of link

$a_{0 t}=$ initial position of $a_{i}$

$a_{0(i+1)}=$ initial position of $a_{1+1}$

Table 3. Reference orientation of measurement axes

\begin{tabular}{llllll}
\hline & & & \multicolumn{3}{c}{ Direction cosines } \\
\cline { 5 - 6 } Joint & $\begin{array}{c}\text { Measured } \\
\text { Motion }\end{array}$ & Axis & $x$ & $y$ & $z$ \\
\hline Hip & Flexion & $\mathbf{u}_{20}$ & 0 & 0 & 1.0000 \\
Hip & Adduction & $\mathbf{v}_{20}$ & 1.0000 & 0 & 0 \\
Knee & Flexion & $\mathbf{u}_{20}$ & 0 & -0.1132 & 0.9936 \\
Ankle & Flexion & $\mathbf{u}_{40}$ & -0.3420 & 0 & 0.9397 \\
\hline
\end{tabular}


Position analysis should begin at the reference member of which absolute motion is defined in terms of absolute measured angles and coordinates.

\section{Velocity analysis}

A numerical harmonic differentiation is used to compute the first and second derivatives of measured displacements. The velocity analysis is developed in similar fashion to that of position. Here the absolute angular velocity $\dot{\theta}_{i}$ of link $i$ is expressed in terms of the known absolute angular velocity $\dot{\theta}_{i-1}$ of member $i-1$ and the derivatives of the relative rotations $\phi_{\vec{r}} \phi_{m}$ and $\phi_{\vec{v}}$ measured along the instrument axes. Furthermore, the first and second derivatives rotations are also known through differentiation. For convenience the intermediate links of the measuring device are introduced as members $i^{\prime}$ and $i^{\prime \prime}$ (Fig. 12). For example, $\dot{\theta}_{i}^{\prime}$ refers to the absolute angular velocity of link $i$. This velocity is given by

$$
\dot{\theta}_{i}=\dot{\theta}_{i-1}+\dot{\phi}_{w}
$$

where

$\dot{\theta}_{i-1}=$ absolute angular velocity of number $i-1$

$\dot{\phi}_{v}=$ angular velocity of $i$ relative to $i-1$ which is $=\dot{\phi}_{u} u$.

Similar expressions are written for member $i^{\prime \prime}$ and $i^{\prime}$ as follows

$$
\begin{aligned}
& \dot{\theta}_{i}^{\prime}=\dot{\theta}_{i}+\dot{\phi}_{v}, \\
& \dot{\theta}_{i}=\dot{\theta}_{i}^{\prime}+\dot{\phi}_{n} .
\end{aligned}
$$

The substitution of equations (A8) and (A9) into (A10) yields

$$
\dot{\theta}_{i}=\dot{\theta}_{i-1}+\dot{\phi}_{u}+\dot{\phi}_{i}+\dot{\phi}_{w}
$$

The velocity of a point $c_{l}$ on member $i$ can be written in terms of the velocity of a point $a_{i}$ if the position vector $r=c_{i}$ $-a_{i}$ is difierentiated as follows:

$$
\dot{\mathbf{r}}=\dot{\boldsymbol{\theta}}_{\boldsymbol{i}} \times \mathbf{r} .
$$

The vector cross product operation $\dot{\theta}_{i} \times$ can be replaced with a matrix product having the following form:

$$
\dot{\mathbf{r}}=\left[W_{i}\right] \mathbf{r} \text {, }
$$

where

$$
\left[W_{i}\right]=\left[\begin{array}{ccc}
0 & -\dot{\theta}_{3} & \dot{\theta}_{2} \\
\dot{\theta}_{3} & 0 & -\dot{\theta}_{1} \\
-\dot{\theta}_{2} & \dot{\theta}_{1} & 0
\end{array}\right] \text {, }
$$

and $\dot{\theta}_{1}, \dot{\theta}_{2}, \dot{\theta}_{3}=$ components of the absolute angular velocity $\dot{\theta}_{i}$. The matrix $\left[W_{i}\right]$ is called the angular velocity matrix of member $i$; it is a convenient equivalent to the cross product operation $\dot{\theta}_{i} \times$ (see Pipes, 1963, pp. 163-174). According to equation (A13), the velocity of the center of mass $\dot{c}_{i}$ has the following form:

$$
\dot{\mathbf{c}}_{i}=\dot{\mathbf{a}}_{i}+\left[W_{i}\right]\left(\mathbf{c}_{0 i}-\mathbf{a}_{0 i}\right) \text {. }
$$

The velocity of the distal joint $\dot{\mathbf{m}}_{i+1}$ is obtained in a similar form to equation (A15) if $c_{i}$ is replaced by $a_{i+1}$ in this equation. Because the velocity of a point on the reference member and its absolute angular velocities are computed from measured displacements, the velocity analysis starts with the computation of this member's center of mass and angular velocities in terms of the computed derivatives.

Acceleration analysis

This analysis follows the general approach in the previous section. The absolute angular acceleration of members $i^{\prime}, i^{\prime \prime}$ and $i$ are expressed as

$$
\begin{aligned}
& \ddot{\theta}_{i}=\ddot{\theta}_{i-1}+\dot{\theta}_{i-1} \times \dot{\theta}_{i}^{\prime}+\ddot{\phi}_{u}, \\
& \ddot{\theta}_{i}^{\prime}=\ddot{\theta}_{i}^{\prime}+\dot{\theta}_{i}^{\prime} \times \dot{\theta}_{i}^{\prime}+\ddot{\phi}_{i}
\end{aligned}
$$

and

$$
\ddot{\theta}_{i}=\ddot{\theta}_{i}^{\prime}+\dot{\theta}_{i}^{\prime \prime} \times \dot{\theta}_{i}+\ddot{\phi}_{w}
$$

Substituting from equation (A8) into (A16) for $\dot{\theta}_{i}$ gives

$$
\ddot{\theta}_{i}=\ddot{\theta}_{i-1}+\dot{\theta}_{i-1} \times \dot{\phi}_{v}+\ddot{\phi}_{u}
$$

Successive substitutions form equations (A17) and (A19) into (A18) eliminates the intermediate quantities and results in an expression for the absolute angular acceleration $\theta_{i}$ in terms of measured or computed quantities:

$$
\begin{aligned}
\ddot{\theta}_{i}= & \ddot{\theta}_{i-1}+\ddot{\phi}_{u}+\ddot{\phi}_{v}+\ddot{\phi}_{w}+\dot{\theta}_{i-1} \times \dot{\phi}_{w} \\
& +\left(\dot{\theta}_{i-1}+\dot{\phi}_{v}\right) \times \dot{\phi}_{v} \\
& +\left(\dot{\theta}_{i-1}+\dot{\phi}_{u}+\dot{\phi}_{v}\right) \times \dot{\phi}_{w}
\end{aligned}
$$

Differentiation of equation (A12) produces the acceleration,

$$
\ddot{\mathbf{r}}=\ddot{\theta}_{i} \times \mathbf{r}+\dot{\boldsymbol{\theta}}_{i} \times\left(\dot{\boldsymbol{\theta}}_{i} \times \mathbf{r}\right) .
$$

Again the vector cross product operations are replaced with matrix operations as follows:

$$
\ddot{\mathbf{r}}=\left\{\left[\alpha_{i}\right]+\left[W_{i}\right]\left[W_{i}\right]\right\} \mathbf{r},
$$

where

$$
\left[\alpha_{i}\right]=\left[\begin{array}{ccc}
0 & -\ddot{\theta}_{3} & \ddot{\theta}_{2} \\
\ddot{\theta}_{3} & 0 & -\ddot{\theta}_{1} \\
-\ddot{\theta}_{2} & \ddot{\theta}_{1} & 0
\end{array}\right] \text {. }
$$

and $\ddot{\theta}_{1}, \ddot{\theta}_{2}, \ddot{\theta}_{3}=$ components of $\ddot{\theta}_{i}$.

The matrix $[A]$ is defined as,

$$
[\mathrm{A}]=\left[\alpha_{i}\right]+\left[W_{i}\right]\left[W_{i}\right] \text {. }
$$

If the matrix operations on the right side of equation (A23) are carried out, matrix [A] takes the following form:

$$
\left[A_{i}\right]=\left[\begin{array}{ccc}
-\left(\dot{\theta}_{2}^{2}+\dot{\theta}_{3}^{2}\right) \dot{\theta}_{1} \dot{\theta}_{2}-\ddot{\theta}_{3} & \dot{\theta}_{1} \dot{\theta}_{3}+\ddot{\theta}_{2} \\
\dot{\theta}_{1} \dot{\theta}_{2}+\ddot{\theta}_{3} & -\left(\dot{\theta}_{3}^{2}+\dot{\theta}_{1}^{2}\right) & \dot{\theta}_{3} \dot{\theta}_{4}-\ddot{\theta}_{1} \\
\dot{\theta}_{1} \dot{\theta}_{3}-\ddot{\theta}_{2} & \dot{\theta}_{3} \dot{\theta}_{2}+\ddot{\theta}_{1} & -\left(\dot{\theta}_{1}^{2}+\dot{\theta}_{2}^{2}\right)
\end{array}\right],
$$

Making the use of equations (A22) and (A23), the acceleration of the center of mass $\ddot{\mathbf{c}}_{i}$ can be expressed in terms of the known quantities as:

$$
\ddot{\mathbf{c}}_{i}=\ddot{\mathbf{a}}_{i}+\left[\mathrm{A}_{i}\right]\left(\mathbf{c}_{0 i}-\mathbf{a}_{0 i}\right)
$$

